\title{
Plasmalemmal Vesicle-Associated Protein Is Associated with Endothelial Cells Sprouting from the Peribiliary Capillary Plexus in Human Cirrhotic Liver
}

\author{
Hiroaki Yokomori ${ }^{\mathrm{a}}$ Wataru Ando ${ }^{\mathrm{b}}$ Masaya Oda ${ }^{\mathrm{c}}$

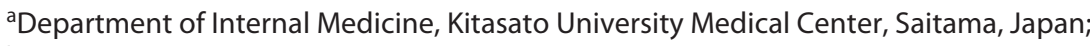 \\ bDepartment of Pharmaceutical Science, Kitasato University, Tokyo, Japan; 'The Chunichi Newspapers, \\ The Main Tokyo Clinic, Tokyo, Japan
}

\section{Keywords}

Plasmalemmal vesicle-associated protein - Peribiliary

capillary plexus · Caveolin-1 - Liver cirrhosis .

Immunoelectron microscopy

\begin{abstract}
Introduction: Plasmalemmal vesicle-associated protein (PLVAP) is an endothelial-specific integral membrane glycoprotein that localizes to caveolae and fenestrae in animal models; however, little is known about PLVAP in endothelial cells (ECs) in hepatic sinusoids during liver cirrhosis (LC). Here, we aimed to elucidate PLVAP localization and expression in the human liver during LC progression. Methods: PLVAP protein expression was detected in specimens from normal control livers and hepatitis C-related cirrhotic livers using immunohistochemistry, Western blotting, and immunoelectron microscopy. Results: PLVAP mainly localized to the peribiliary capillary plexus (PCP) and was rarely observed in hepatic artery branches and portal venules in control tissue, but was aberrantly expressed in capillarized sinusoids and proliferated capillaries in fibrotic septa within cirrhotic liver tissue. Ultrastructural analysis indicated that PLVAP localized to thin ECs in some caveolae, whereas
\end{abstract}

karger@karger.com www.karger.com/jvr

Karger"

BOPEN ACCESS
(C) 2021 The Author(s)

Published by S. Karger AG, Basel

This is an Open Access article licensed under the Creative Commons Attribution-NonCommercial-4.0 International License (CC BY-NC) (http://www.karger.com/Services/OpenAccessLicense), applicable to the online version of the article only. Usage and distribution for commercial purposes requires written permission.
PLVAP localized primarily to caveolae-like structures and proliferative sinusoid capillary EC vesicles in cirrhotic liver tissue. Western blot analysis confirmed that PLVAP was overexpressed at the protein level in advanced cirrhotic liver tissue. Conclusion: PLVAP was strongly expressed in the caveolae of proliferated capillaries directly connected with sinusoids linked with the PCP, suggesting that it plays a role in angiogenesis and sinusoidal remodeling in LC.

(C) 2021 The Author(s)

Published by S. Karger AG, Basel

\section{Introduction}

Plasmalemmal vesicle-associated protein (PLVAP, also known as MECA32 and PV-1) is an endothelial-specific, cationic, integral membrane glycoprotein that was the first molecular component to be identified in fenestrae. As well as being essential for the development of fenestral and stomatal diaphragms in endothelial cells (ECs) $[1,2]$, PLVAP also regulates basal permeability, leukocyte migration, and angiogenesis [2]. Previous studies have suggested that PLVAP may be induced during hypoxia, since it is upregulated in patients with acute 
ischemia [2, 3]. Moreover, studies have demonstrated that PLVAP is strictly localized to endothelial caveolae isolated from rat lungs using anti-caveolin-1 (CAV-1) antibodies $[2,4,5]$.

Liver sinusoidal endothelial cells (LSECs) differ from ECs in other capillaries as they contain fenestrations and lack a basal lamina [6]. However, LSECs lose their fenestrations and develop an organized basement membrane during liver cirrhosis (LC) [7], while the development of fibrotic scars is invariably accompanied by intense vascular proliferation and proliferative arterial capillary plexuses [8]. Interestingly, it has been shown that the number of LSEC fenestrations is markedly reduced in PLVAPdeficient mice [9].

The peribiliary capillary plexus (PCP) is a network of capillaries derived from branches of the hepatic artery that surround the intrahepatic bile ducts and drain into the portal veins or hepatic sinusoids. Although the PCP surrounding the interlobular bile ducts has only 1 layer and is characterized by scattered capillaries, the PCP surrounding large bile ducts has 2 layers and is characterized by chain-like capillaries $[10,11]$. However, analysis using the endothelial marker anti-CD34 antibody revealed that the number of PCP capillaries is significantly higher in cirrhotic livers than in normal livers [12].

Although the molecular mechanisms underlying angiogenesis during the progression of chronic viral hepatitis have not yet been fully elucidated, accumulating evidence has suggested that angiogenesis plays an important role in this process $[13,14]$. For instance, the activation of vascular endothelial growth factor receptor (VEGFR)-2 in human umbilical vein endothelial cells stimulated with VEGF, the primary regulator of PLVAP, has been shown to increase PLVAP mRNA and protein expression and thereby increase vascular permeability and angiogenesis [2-4]. In this study, we describe the localization of PLVAP in sinusoids and the portal tract as well as its effect on hepatitis C-related LC.

\section{Materials and Methods}

\section{Antibodies}

The following antibodies were used in this study: rabbit antiPLVAP (NBP1-83911; Novus Biol., Littleton, CO, USA) and rabbit anti-CAV-1 (610060; BD, Franklin Lakes, NJ, USA). To avoid background signals, heat-mediated antigen retrieval was conducted using an autoclave for $15 \mathrm{~min}$ at $120^{\circ} \mathrm{C}$, followed by staining with N-Histofine ${ }^{\circledR}$ Simple Stain AP, MULTI (Nichirei, Tokyo, Japan). Bound antibodies were visualized using 3,3'-diaminobenzoic tetrahydrochloride (D-8001; Sigma-Aldrich, St. Louis, MO, USA) and $\mathrm{H}_{2} \mathrm{O}_{2}$ solution.

\section{Samples}

To detect changes in CAV-1 and PLVAP localization and expression during LC progression, we studied 3 groups of tissues, as follows: group 1, normal control liver tissue; group 2, liver tissue from patients with early-stage cirrhosis; and group 3, liver tissue from patients with advanced-stage cirrhosis. As controls (group 1), wedge biopsy specimens were obtained from the normal areas of the livers from 6 patients (5 males and 1 female; aged 64-83 years, mean 76.4 years) who underwent surgical resection for metastatic liver carcinoma (colon carcinoma in 2 patients, gastric carcinoma in 1 patient, sclerosed hemangioma in 1 patient, and hepatocellular carcinoma [HCC] in 2 patients). Early cirrhotic liver specimens (group 2) were obtained from grossly cirrhotic areas surgically resected from 7 patients ( 4 males and 3 females; aged 58-76 years, mean 70.8 years) who underwent hepatectomy for HCC with concurrent hepatitis $\mathrm{C}$ virus-related cirrhosis and were classified as Child-Pugh grade A. Advanced cirrhotic liver tissues (group 3) were obtained from 5 autopsies performed within $3 \mathrm{~h}$ after death ( 3 males and 2 females; aged $72-78$ years, mean 74.4 years). All cases were diagnosed with HCC and concurrent hepatitis C-related cirrhosis and were classified as Child-Pugh grade C. This study was approved by the Ethics Committee of the Kitasato University Medical Center (No. 25-16).

\section{Methods}

Immunohistochemistry (IHC), immunoelectron microscopy (IEM), and Western blotting were conducted as described previously [15] with minor modifications. CAV-1 and PLVAP in control and Child-Pugh grade A cirrhotic liver samples were analyzed using immunogold-silver enhancement, as described previously [15].

\section{Immunohistochemistry}

Liver tissues (approximately $3 \times 2 \times 1 \mathrm{~cm}$ ) were fixed in formalin, embedded in paraffin, and then serial sections $(4 \mu \mathrm{m})$ were deparaffinized and rehydrated by immersion in xylene and a graded alcohol series. Endogenous peroxidase activity was quenched by incubation in $0.3 \%(\mathrm{v} / \mathrm{v}) \mathrm{H}_{2} \mathrm{O}_{2}$ in methanol for $30 \mathrm{~min}$ at room temperature (RT). Heat-mediated antigen retrieval was performed using $10 \mathrm{~mm}$ citrate buffer ( $\mathrm{pH}$ 6.0) in an autoclave for $15 \mathrm{~min}$ at $120^{\circ} \mathrm{C}$. Sections were blocked with $10 \%$ normal goat serum (Vector Laboratories, Burlingame, CA, USA), incubated for 90-120 min with primary antibodies in $2 \%$ serum, and then incubated overnight at $4^{\circ} \mathrm{C}$ with anti-CAV-1 (1:250 dilution) or -PLVAP (1:400 dilution) rabbit polyclonal antibodies. The sections were then incubated with N-Histofine ${ }^{\circledR}$ Simple Stain MAX PO (Nichirei, Tokyo, Japan) at RT for $30 \mathrm{~min}$. After repeated washing with PBS, the sections were reacted with diaminobenzidine containing $0.01 \%$ $\mathrm{H}_{2} \mathrm{O}_{2}$ and counterstained with hematoxylin for light microscopy $[16,17]$.

\section{Computer-Assisted Morphometric Analysis}

Morphometric variables were determined by labeling sections with anti-PLVAP antibodies. Three periportal regions and regenerative fibrotic areas were assessed separately in each liver tissue section. In brief, immunostained sections were scanned under a light microscope at low magnification $(\times 4.2)$, and vessels in each region were enumerated at a high magnification $\left(\times 20 ; 0.304 \mathrm{~mm}^{2} /\right.$ 
Fig. 1. Immunohistochemical analysis of CAV-1 and PLVAP in control and cirrhotic liver specimens. a-d Normal control liver (zone 1). a, b CAV-1 localized mainly to capillary vessels was detected in large vessels and portal venules in the portal tract and was partly detected in sinusoids. c, d PLVAP was expressed in vascular and portal vein tissues. CAV-1 immunoperoxidase-positive substrates were weakly detected around zone 1 and more frequently detected around zone 3 of the hepatic sinusoidal linings of normal liver specimens. a, c Low magnification. b, d High magnification. Arrows denote reaction products and vessels of PLVAP. e-h Child-Pugh grade A cirrhotic liver. e, f CAV-1 reaction products partially localized to hepatic sinusoids and proliferative arterial capillaries directly connected with sinusoids to generate hepatic nodules. g, h PLVAP immunostaining was mainly detected in proliferated capillary arteries and sinusoidal lining cells in the peripheral regions of nodules. $\mathrm{P}$ denotes the portal tract. C denotes the central vein. e, $\mathbf{g}$ Low magnification. f, h High magnification. Arrowheads denote reaction products and capillaries of PLVAP. i-I ChildPugh grade $\mathrm{C}$ cirrhotic liver. $\mathbf{i}$, $\mathbf{j}$ Reaction products indicating CAV-1 expression were observed on proliferated arterial capillaries in the peripheral region of fibrous septa and aberrantly on sinusoids in regenerative nodules surrounded by broad fibrous septa, indicating hepatic sinusoid capillarization. $\mathbf{k}$, I PLVAP immunoreactivity was mainly enhanced on sinusoidal lining cells in regenerated nodules and in the peripheral regions of nodules and fibrous septa. i, k Low magnification. j, I High magnification. Arrowheads denote reaction products and capillaries of CAV-1 and PLVAP. PLVAP, plasmalemmal vesicleassociated protein; CAV-1, caveolin-1.
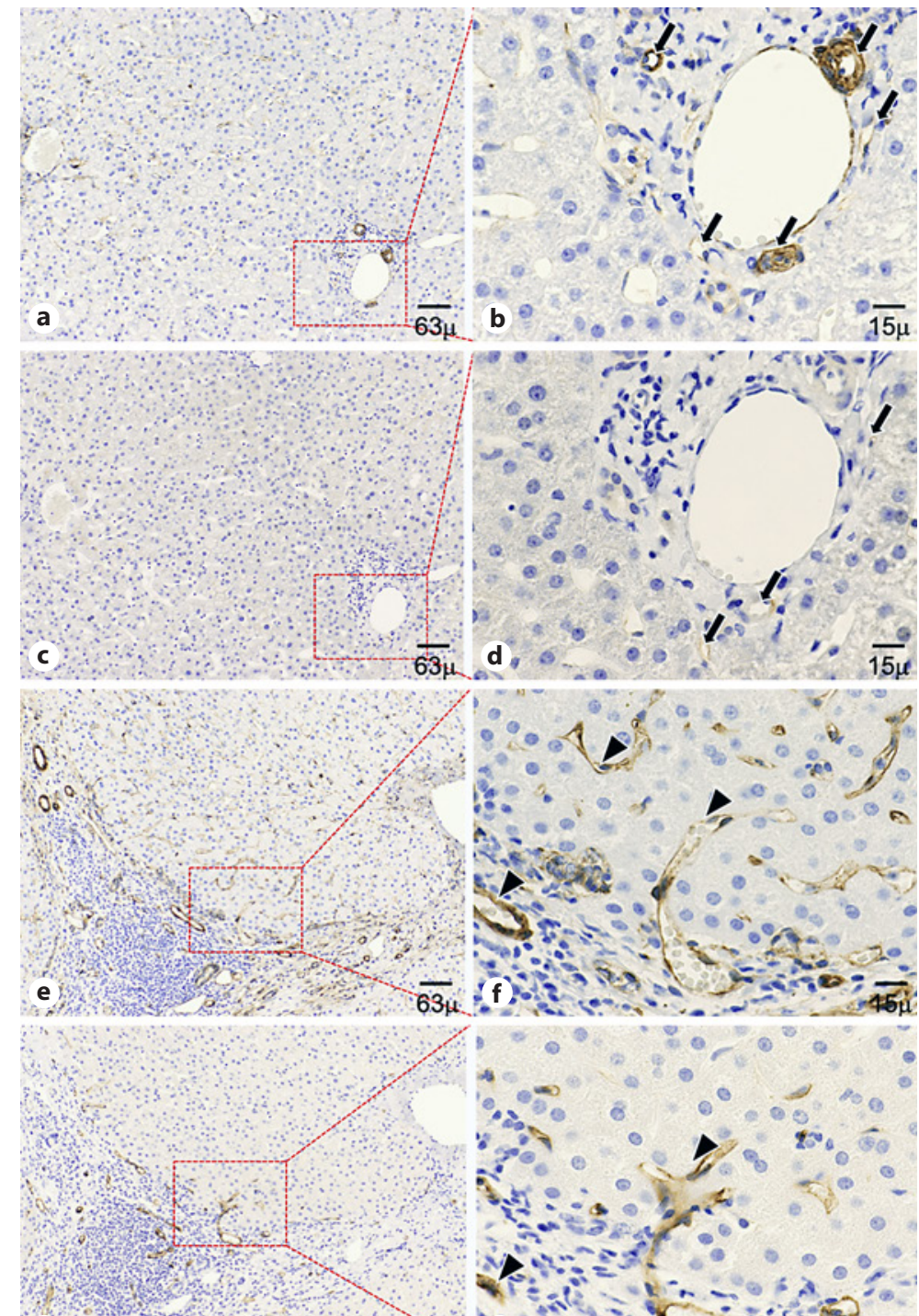

$\mathbf{g}$
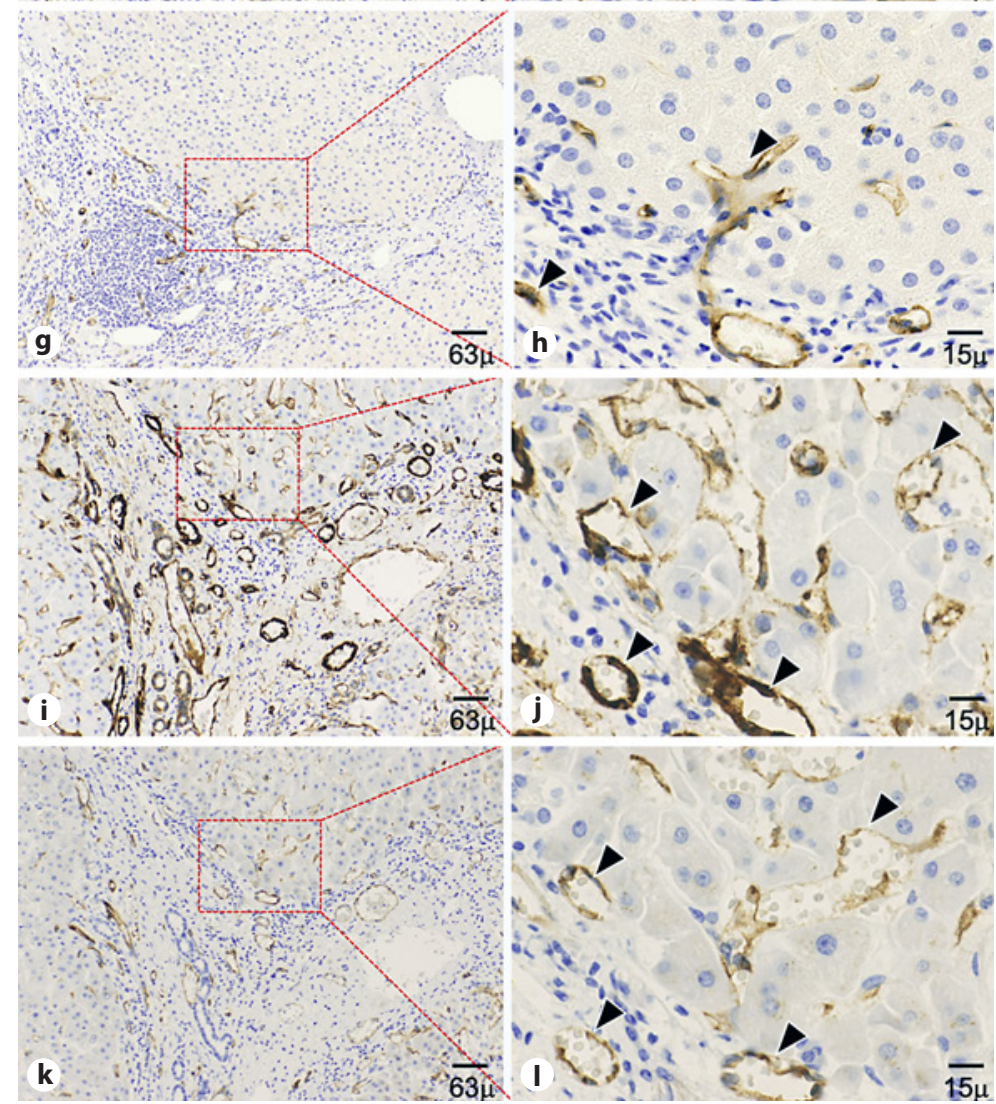


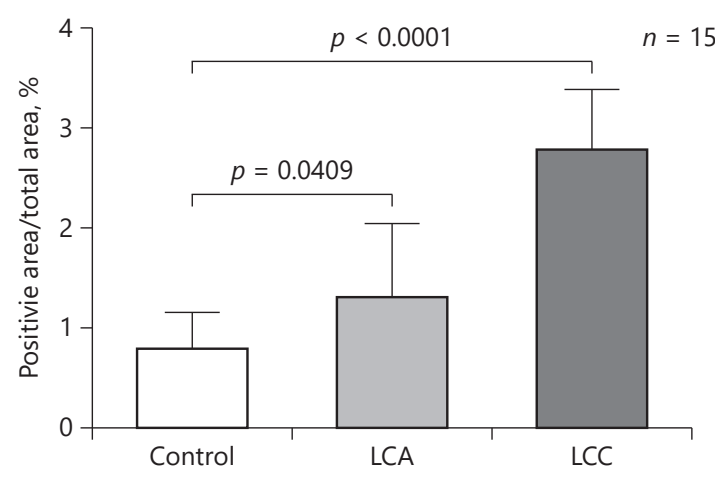

Fig. 2. Morphometric analysis. PLVAP was highly expressed in early-stage LC and was expressed at much lower levels in control liver tissues. Fifteen randomly selected nonserial liver specimens were analyzed from hepatitis $\mathrm{C}$-positive unaffected cases $(N=4)$, cases with Child-Pugh grade A bridging fibrosis (LCA, $N=4)$, and cases with Child-Pugh grade $\mathrm{C}$ cirrhosis (LCC, $N=4$ ). Statistical analysis was performed using one-way ANOVA with Dunnett's post hoc multiple comparisons test. $N$, cases; $n$, liver specimens; PLVAP, plasmalemmal vesicle-associated protein; LC, liver cirrhosis.

field). The stained sections were also visualized under a standard light microscope (magnification, $\times 20$; Olympus, Tokyo, Japan). At least 5 images were captured using a digital camera $(3,072 \times 2,304$ size, 32-bit) to represent the entire periportal area and saved in JPEG format, during which the captured images were converted into points or pixels according to red tone to assess PLVAP staining in capillary ECs. The statistical significance of differences in PLVAP expression between control (periportal region or pericentral regions) and LC samples was assessed using the Wilcoxon/ Kruskal-Wallis test with Bonferroni's post hoc test. Statistical analyses were conducted using EZR software (version 1.32; Saitama Medical Center, Jichi Medical University, Shimotsuke, Japan) and $\mathrm{R}$ (version 2.2.0; The R Foundation for Statistical Computing, Vienna, Austria; http://www.R-project.org/). $p$ values $<0.05$ were considered significant. Data are expressed as the mean \pm standard error of the mean.

\section{Western Blot Analysis}

All patient samples $\left(1.5-\mathrm{cm}^{3}\right.$ sections, nonrepetitions in 4 subjects) were homogenized in 10 volumes of homogenization buffer (20 $\mu \mathrm{M}$ Tris- $\mathrm{HCl}$ ( $\mathrm{pH} 7.5), 5$ mM $\mathrm{MgCl}_{2}, 0.1$ mM PMSF, $20 \mu \mathrm{M}$ pepstatin $\mathrm{A}$, and $20 \mu \mathrm{M}$ leupeptin) using a polytron homogenizer at setting 7 for $90 \mathrm{~s}$. The obtained membrane proteins were subjected to immunoblotting. In brief, proteins $(30 \mu \mathrm{M} / \mathrm{mL})$ were separated by SDS-PAGE and transferred onto polyvinylidene difluoride membranes (NEN Life Science Products). The membranes were blocked with 5\% (w/v) dried milk in PBS for $30 \mathrm{~min}$ and incubated with anti-PLVAP antibodies diluted $1: 1,000$ in PBS with $0.1 \%$ Tween 20. After the blots had been washed, they were incubated for $1 \mathrm{~h}$ at RT with horseradish peroxidase-conjugated goat antirabbit immunoglobulin diluted 1:20,000 (Santa Cruz Biotechnol- ogy). Protein bands were detected using an enhanced chemiluminescence detection system (ECL Plus; Amersham Biosciences, Uppsala, Sweden). After the nitrocellulose sheets had been exposed to ImageQuant LAS-4000 mini (Fujifilm Wako, Inc., Osaka, Japan), $\beta$-actin (Cosmo, Tokyo, Japan) was used as a loading control for Western blot analysis $[16,17]$.

\section{Immunohistological Quantification of PLVAP Expression by} Western Blot Analysis

The expression levels of PLVAP and $\beta$-actin were determined by Western blotting. Expression of PLVAP and $\beta$-actin was pixeled, and the ratio was compared using ImageJ $1.52 \mathrm{a}$ software (NIH, Bethesda, MD, USA) [18].

\section{Immunogold-Silver Staining for Electron Microscopy}

We used a modified perfusion-fixation method to fix organelle and molecule structures in their in vivo positions [16]. In brief, wedge biopsies (approximately $3 \times 1 \times 1 \mathrm{~cm}$ ) were obtained from the margin of the liver lobe as soon as the operator had access to control or Child-Pugh A cirrhotic livers and immediately transferred to a container filled with PBS ( $\mathrm{pH} \mathrm{7.4)} \mathrm{at} 37^{\circ} \mathrm{C}$. Using forceps, the corner of each biopsy sample was injected from multiple sides with a periodate-lysine-paraformaldehyde fixative using a 26-G syringe in a perfusion-mediated manner in a Petri dish filled with saline until the tissue became discolored and hardened. After perfusion and incubation with periodate-lysine-paraformaldehyde overnight at $4^{\circ} \mathrm{C}$, semi-thin $1-\mathrm{mm}$ sections were prepared and treated as follows: (1) immersed 3 times for $15 \mathrm{~min}$ in $0.01 \%$ PBS (pH 7.4), (2) incubated with anti-PLVAP antibodies diluted $1: 400$ in $0.01 \mathrm{M}$ PBS containing $1 \%$ bovine serum albumin overnight at $4^{\circ} \mathrm{C}$ in a moist chamber, (3) treated with PBS 3 times for $15 \mathrm{~min}$, (4) incubated with 1.4 colloidal gold-conjugated anti-rabbit IgG antibodies (Nanogold ${ }^{\circledR}$; Nanoprobes, Yaphank, NY, USA) diluted 1:40 for $40 \mathrm{~min}$, and (5) physically developed using a silver enhancement kit (Nanoprobe Silver Enhancement Kit; Nanoprobes) $[16,17]$. The liver sections were then washed 3 times with $0.01 \%$ PBS, fixed in $1.5 \%$ glutaraldehyde with $0.01 \%$ PBS (pH 7.4) for $1 \mathrm{~h}$ at $4^{\circ} \mathrm{C}$, incubated with a graded ethanol series, and postfixed with $2 \%$ osmium tetroxide in $0.01 \%$ PBS ( $\mathrm{pH} 7.4$ ). After embedding in Epon, ultrathin sections were cut using an LKB ultra microtome with a diamond knife, stained with uranyl acetate, and observed under a transmission electron microscope (JEM-1200 EX; JEOL, Tokyo, Japan) with an $80-\mathrm{kV}$ acceleration voltage [16, $19]$.

\section{Results}

\section{IHC Analysis of CAV-1 and PLVAP in Human}

Control and Cirrhotic Liver Tissues

CAV-1 is expressed in the vascular walls and portal vein tissues. Here, we detected CAV-1 immunoperoxidase-positive substrates around zone 1 and more frequently around zone 3 in the hepatic sinusoidal lining of normal control liver specimens (Fig. 1a, b). In these tissues, PLVAP was mainly localized to capillary venules but was also detected on large vessels in the portal tract and 


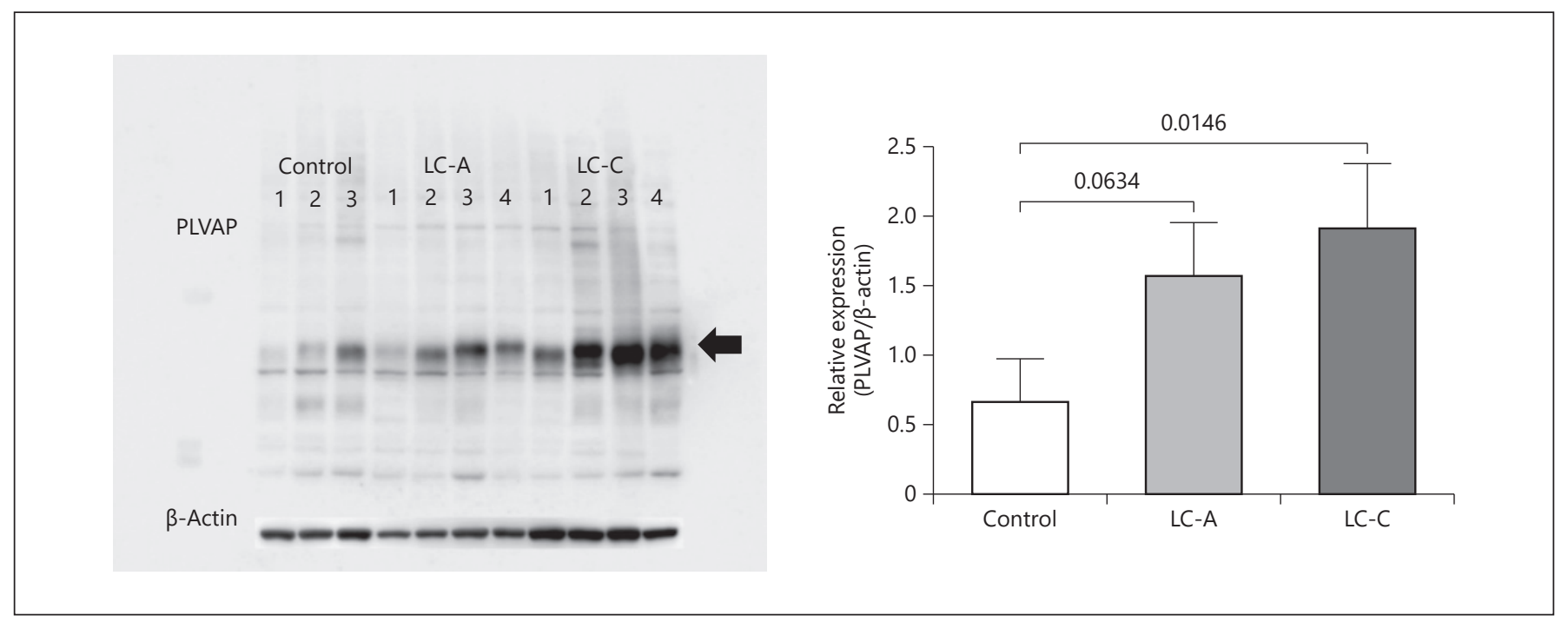

Fig. 3. Western blot analysis of PLVAP expression. PLVAP was overexpressed in the liver specimens of patients with advanced hepatitis C-related LC. Arrowheads indicate the band corresponding to PLVAP. Control: normal control liver specimens; LCA, Child-Pugh grade A cirrhotic liver specimens; LCC, Child-Pugh grade C cirrhotic liver specimens; PLVAP, plasmalemmal vesicle-associated protein; LC, liver cirrhosis.

partially in hepatic sinusoids around the portal tract (Fig. 1c, d). In view of the large portal tracts, PLVAP was localized on scattered capillaries around the PCP bile ducts (online suppl. Fig. 1a, b; for all online suppl. material, see www.karger.com/doi/10.1159/000516923).

In Child-Pugh grade A cirrhotic liver tissue, CAV-1 immunostaining was mainly detected in proliferative arterial capillaries and sinusoidal lining cells in the peripheral regions of nodules (Fig. 1e, f). Consistently, PLVAP reaction products were partially localized to hepatic sinusoids and proliferative arterial capillaries that were directly connected with sinusoids in regenerating hepatic nodules (Fig. 1g, h). In Child-Pugh grade $\mathrm{C}$ cirrhotic liver tissue, CAV-1 immunoreactivity was mainly observed on sinusoidal lining cells in regenerated nodules and in the peripheral regions of nodules and fibrous septa (Fig. 1i, j). Reaction products indicating PLVAP expression were also evident on proliferative arterial capillaries in the peripheral region of fibrous septa, and aberrant expression was observed on sinusoids in regenerative nodules surrounded by broad fibrous septa, indicating hepatic sinusoid capillarization (Fig. 1k, 1). The morphometric examination of periportal and fibrotic areas revealed that PLVAP expression was significantly higher in patients with Child-Pugh grade A or C LC than in the control individuals (Fig. 2).

\section{Western Blot Analysis}

To confirm the results of our IHC analysis, we analyzed PLVAP expression in normal and cirrhotic liver tissues using Western blotting. As expected, PLVAP was highly expressed in Child-Pugh grade $\mathrm{C}$ cirrhotic liver tissue and was expressed at much lower levels in control liver tissue (Fig. 3).

\section{Ultrastructural Localization of PLVAP}

To determine whether PLVAP expression was altered in LC, we analyzed its ultrastructural localization using IEM. In control liver tissue, immunogold staining for PLVAP immunoreactivity was detected at low levels in ECs in arterial vessel branches (Fig. $4 \mathrm{a}, \mathrm{b}$ ) and LSECs on hepatic sinusoids (Fig. 4c, d). In addition, PLVAP was present in some caveolae and spikes on the surface of true capillary ECs in scattered capillaries (Fig. 4e, f), while gold particles indicating CAV-1 were detected in caveolae and on some vesicles of capillary ECs in normal control liver tissue (online suppl. Fig. 2a, b).

In Child-Pugh grade A cirrhotic liver tissue, PLVAP was localized to the ECs of artery branches (Fig. 5a, b) and was primarily localized to caveolae-like structures and the vesicles of proliferative capillary ECs with adhesive contact to the EC membrane (Fig. 5c, d). Moreover, gold particles indicating CAV-1 were observed on the caveolae of capillary ECs in cirrhotic liver tissue (online suppl. Fig. 2c, d). 


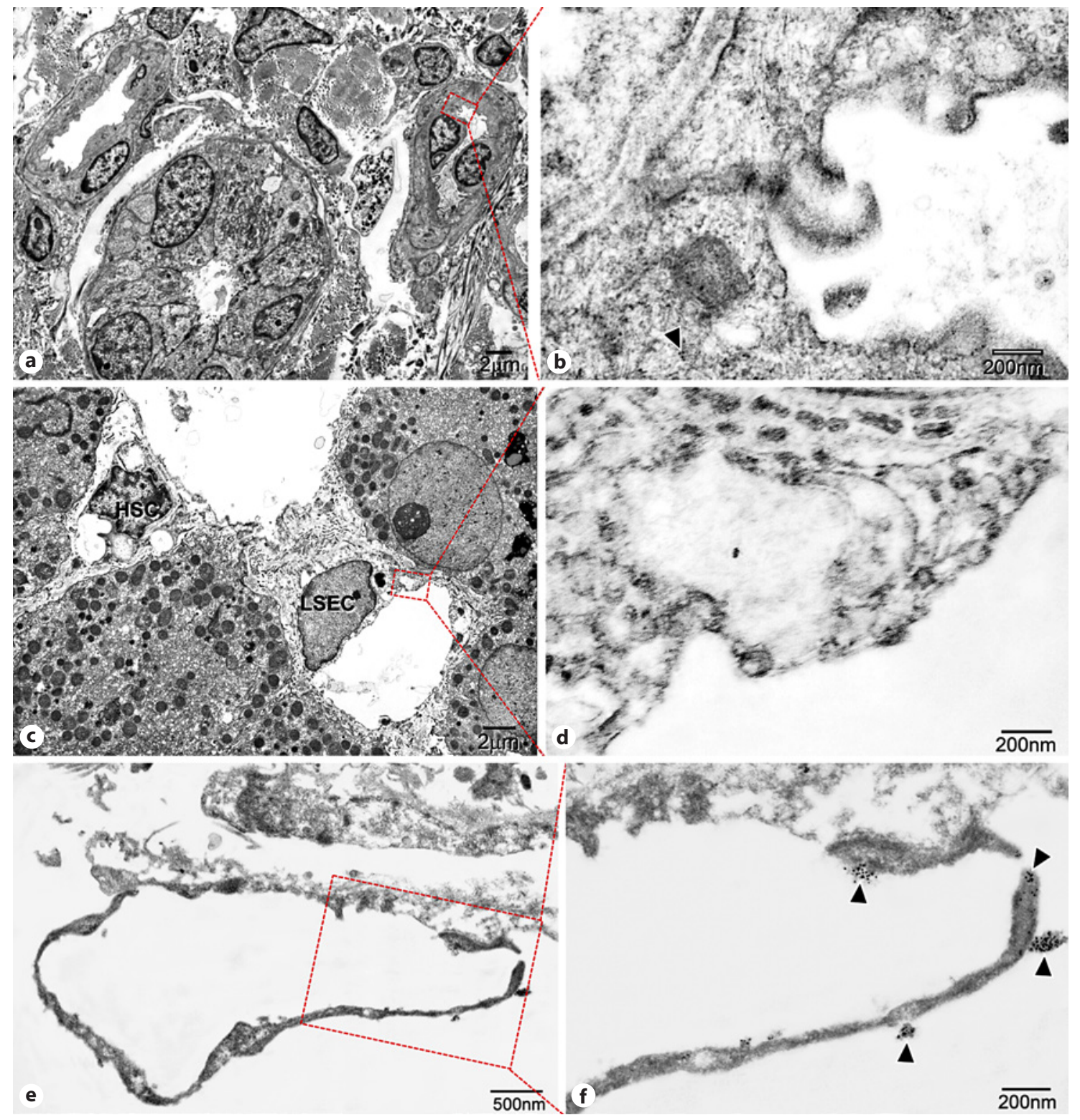

Fig. 4. IEM analysis of PLVAP in normal control liver specimens. a, b Little labeling was observed on the surface of capillary ECs in the branches of arteries. c, $\mathbf{d}$ Little labeling was observed on the plasma membrane and vesicles of LSECs. e, $\mathbf{f}$ Labeling was detected on some caveolae and microspikes on the surface of thin capillary ECs. a, $\mathbf{c}$, e Low magnification. $\mathbf{b}, \mathbf{d}, \mathbf{f}$ High magnification. e denotes ECs. Arrowheads denote reaction products. PLVAP, plasmalemmal vesicle-associated protein; IEM, immunoelectron microscopy; ECs, endothelial cells; LSECs, liver sinusoidal endothelial cells.

\section{Discussion}

In this study, we investigated the localization and expression of PLVAP in the human liver as well as changes during LC progression. Our IHC results revealed that PLVAP is mainly localized to proliferative capillary ECs in fibrotic septa and hepatic sinusoids in cirrhotic livers, while IEM confirmed that PLVAP is present in the caveolae of proliferative capillary ECs.

Previous studies have reported that PLVAP is expressed in vascular ECs in HCC but not in the ECs of liver sinusoids, central veins, the portal vein, or hepatic arterioles [20] and is not notably expressed in the ECs of large vessels, except for the endocardial lining of cardiac 


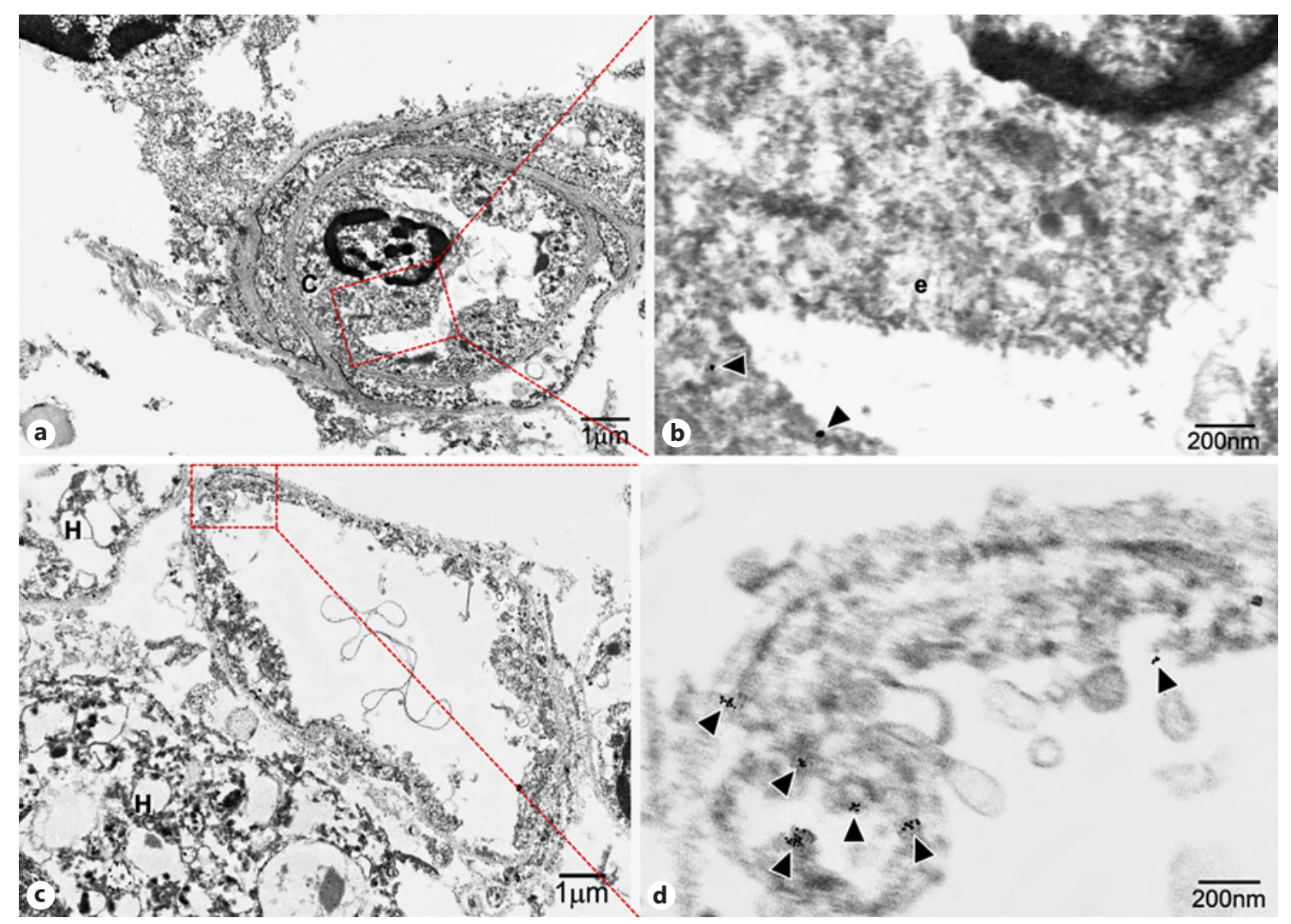

Fig. 5. IEM analysis of PLVAP in cirrhotic liver specimens. a, b Little labeling was observed on the surface of capillary ECs in artery branches. c, d Labeling was detected in the caveolae of some ECs in capillarized hepatic sinusoids. a, c Low magnification. b, d High magnification. c denotes capillary ECs. Arrowheads denote reaction products. PLVAP, plasmalemmal vesicle-associated protein; IEM, immunoelectron microscopy; ECs, endothelial cells.

chambers [2]. Here, we found that CAV-1 localized to caveolae in ECs from arterial vessel branches in both control and cirrhotic liver specimens, while PLVAP was also partly detected on these ECs.

Interestingly, PLVAP was aberrantly expressed on ECs in the regenerative nodules of cirrhotic liver specimens, which may be associated with capillarization and remodeling in this region. However, PLVAP was also aberrantly detected on periportal LSECs corresponding to capillarized sinusoids and on proliferative arterial capillaries opening into sinusoids in regenerating hepatic nodules. Previously, we reported that aquaporin 1 is related to the proliferation of arterial capillaries from vessels of fibrotic septa directly connected with sinusoids, which contributes toward microvascular resistance in human LC [21].

Proinflammatory mediators and other nonhypoxic stimuli can elicit an angiogenic response by inducing hypoxia-inducible factor-1 (HIF-1) and HIF-1-dependent transcriptional activity, including VEGF production [22].
VEGFR-2 is the key mediator of VEGF signaling in ECs [23] and localizes to caveolae [24], while studies have shown that treating human umbilical vein endothelial cells with VEGF increases PLVAP mRNA and protein expression by activating VEGFR-2 [4]. Previously, we reported that the immunoreactivity of VEGFR-2-positive blood vessels is considerably higher in cirrhotic liver tissue than in control liver tissue, and that VEGFR-2 is highly expressed on proliferative capillaries in regenerative hepatic nodules as well as being expressed on hepatic sinusoids [24].

Our ultrastructural analysis demonstrated that PLVAP localized to some caveolae and microspike-like structures in true capillary ECs. PLVAP labeling was mostly found to be associated with the neck of the caveolae or their stomatal diaphragms at both fronts of ECs. Very little labeling with PLVAP, if any, was found on the endothelial plasmalemma proper and coated pits using pre-embedding methods. Following postembedding of IEM cryosections, the label was found at comparable frequencies on 
the caveolae at both fronts of ECs as well as on vesicles within the cytoplasm. According to IEM, the localization of PLVAP following the pre-embedding method presumably reflected the higher accessibility from the lumen.

Moreover, PLVAP protein was negatively regulated by VEGF-R2 in the lung of CAV-1, but not CAV-2, null mice. According to relation of CAV-1 and CAV-2, loss of CAV-1 results in loss of CAV-2 by proteolytic degradation as a result of failed heterodimerization with CAV-1. In contrast, the loss of CAV-2 has little effect on CAV-1 protein or caveolae biogenesis. Thus, CAV-1-mediated protein interaction and targeting remains intact in CAV2 null mice, likely explaining the difference in PLVAP protein levels between these 2 genetic models [25]. The intracellular domain of PLVAP consists of 2 short, identical stretches of amino acids, one of which is adjacent to the transmembrane region and contains a putative CAV1-binding domain, whereas the other is located at the extreme $\mathrm{N}$-terminus [26]. The endothelial surface layer significantly affects hemodynamic conditions such as the mechanical stresses acting on red blood cells in microvessels, oxygen transport, vascular control, coagulation, inflammation, and atherosclerosis [1-4, 26].

PLVAP is restricted to the membrane of a subset of ECs in normal microvasculature $[27,28]$ and was the first molecular component of fenestrae to be identified in ECs [13]. Hepatic microcirculatory sites may include various segments of afferent portal venules and hepatic arterioles, sinusoids, and central and hepatic venules. Although direct connections (arterio-portal anastomoses) have been observed in terminal portal venules, the frequency of these connections appears to be species dependent [29]. A previous immunohistochemical study showed that sinusoidal ECs lacking or containing fenestrae are present in zone 1 of the rodent liver and possess characteristics of vascular (capillary) ECs from the portal triad [21,30]. Consistently, IEM analyses performed in this study detected PLVAP on sinusoidal endothelial fenestrae of LSECs.

Proliferating ductules are often observed at the portal tract interface in patients with hepatitis $\mathrm{C}$ and fibrosis and are thought to arise from hepatic progenitor cells (HPCs) [31]. In addition, a significant relationship exists between the periportal bile ductular reaction and hepatic fibrosis in many patients with chronic hepatitis $\mathrm{C}$ infection. Proliferating ductules likely arise from HPCs based on their close proximity to the ductular reaction and its strong correlation with hepatic fibrosis. Interestingly, steatosis and interface hepatitis are both independently associated with an increase in the number of HPCs and the extent of the ductular reaction [32].
The density of the endothelial marker CD34-positive PCPs per interlobular bile duct during prolonged chronic inflammation and portal hypertension may be linked to PCP angiogenesis during LC [12]. Our IHC and IEM analyses indicated that angiogenesis involving sinusoidal ECs differs from angiogenesis involving ECs in systemic vessels. Moreover, we found that PLVAP localized to areas of the PCP and may be linked to the sprouting of capillary ECs from the PCP during the progression of human LC. However, the potential mechanism via which PVLAP functions in hepatic angiogenesis and sinusoidal remodeling has not yet been investigated. Additional studies are needed to investigate whether our findings can be replicated in experimental animals with liver fibrosis. This would lead to the generation of an alternative experimental animal model, which will permit rigorous laboratory studies of the molecules and proteins involved in the complex process of sinusoidal remodeling.

\section{Acknowledgment}

The authors thank Hitoshi Yamazaki and Yoshihito Takahashi (Kitasato University Medical Center) for providing liver specimens, as well as Hajime Imahana (Kitasato University Medical Center) for assistance with the statistical analysis. The authors also thank Mariko Ogi, Natsuki Hata, and Tomoko Yoshii for their technical assistance.

\section{Statement of Ethics}

The subject (patients or his/her guardian) has given written informed consent. This study protocol was approved by the Kitasato University Medical Center Ethics Committee.

\section{Conflict of Interest Statement}

The authors have no conflicts of interest to declare.

\section{Funding Sources}

H.Y. received scholarship support from AbbVie GK, Otsuka Pharmaceutical Co. Ltd., Zeria Pharmaceutical Co., Ltd., and EA Pharma Co., Ltd. None of the study sponsors had any role in the study design, collection, analysis, or interpretation of the data, in the writing of the report, or in the decision to submit the manuscript for publication.

\section{Author Contributions}

H.Y., W.A., and M.O. designed the study. H.Y. and W.A. conducted the experiments. H.Y., W.A., and M.O. wrote the manuscript. 


\section{References}

1 Stan RV, Ghitescu L, Jacobson BS, Palade GE. Isolation, cloning, and localization of rat PV1, a novel endothelial caveolar protein. J Cell Biol. 1999;145:1189-98.

2 Stan RV. Structure of caveolae. Biochim Biophys Acta. 2005;1746(3):334-48.

3 CarsonWalter EB, Hampton J, Shue E, Geynisman DM, Pillai PK, Sathanoori R, et al. Plasmalemmal vesicle associated protein- 1 is a novel marker implicated in brain tumor angiogenesis. Clin Cancer Res. 2005;11:7643-50.

4 Guo L, Zhang H, Hou Y, Wei T, Liu J. Plasmalemma vesicle-associated protein: a crucial component of vascular homeostasis. Exp Ther Med. 2016;12:1639-44.

5 Hnasko R, McFarland M, Ben-Jonathan N. Distribution and characterization of plasmalemma vesicle protein-1 in rat endocrine glands. J Endocrinol. 2002;175:649-61.

6 Wisse E. An electron microscopic study of the fenestrated endothelial lining of rat liver sinusoids. J Ultrastruct Res. 1970;31:125-50.

7 Schaffner F, Popper H. Capillarization of hepatic sinusoids in man. Gastroenterology. 1963;44:239-42.

8 Rappaport AM, MacPhee PJ, Fisher MM, Phillips MJ. The scarring of the liver acini (cirrhosis): tridimensional and microcirculatory consideration. Virchows Arch. 1983;402: 107-37.

9 Herrnberger L, Hennig R, Kremer W, Hellerbrand C, Goepferich A, Kalbitzer HR, et al. Formation of fenestrae in murine liver sinusoids depends on plasmalemma vesicle-associated protein and is required for lipoprotein passage. PLoS One. 2014;9:e115005.

10 Kobayashi S, Nakanuma Y, Matsui O. Intrahepatic peribiliary vascular plexus in various hepatobiliary diseases: a histological survey. Hum Pathol. 1994;25:940-6.

11 Washington $\mathrm{K}$, Clavien PA, Killenberg P. Peribiliary vascular plexus in primary sclerosing cholangitis and primary biliary cirrhosis. Hum Pathol. 1997;28:791-5.

12 Matsunaga Y, Terada T. Peribiliary capillary plexus around interlobular bile ducts in vari- ous chronic liver diseases: an immunohistochemical and morphometric study. Pathol Int. 1999;49:869-73.

13 Medina J, Arroyo AG, Sánchez-Madrid F, Moreno-Otero R. Angiogenesis in chronic inflammatory liver disease. Hepatology. 2004; 39:1185-95.

14 Elpek GÖ. Angiogenesis and liver fibrosis. World J Hepatol. 2015;7(3):377-91.

15 Yokomori H, Ando W, Yoshimura K, Yamazaki H, Takahashi Y, Oda M. Increases in endothelial caveolin-1 and cavins correlate with cirrhosis progression. Micron. 2015;76: 52-61.

16 Yokomori H, Ando W, Yoshimura K, Yamazaki H, Takahashi Y, Oda M. Increases in endothelial caveolin-1 and cavins correlate with cirrhosis progression. Micron. 2015;76: 52-61.

17 Yokomori H, Ando W, Kaneko F, Suzuki H, Igarashi K, Oda M. Autotaxin and vascular endothelial growth factor receptor-2 and -3 are related to vascular development during the progression of chronic viral hepatitis $\mathrm{C}$. APMIS. 2018;126:913-21.

18 Schneider CA, Rasband WS, Eliceiri KW. NIH image to image: 25 years of image analysis. Nat Methods. 2012;9:671-5.

19 Humbel BM, Sibon OC, Stierhof Y-D, Schwarz H. Ultra-small gold particles and silver enhancement as a detection system in immunolabeling and in situ hybridization experiments. J Histochem Cytochem. 1995;43: 735-7.

20 Wang YH, Cheng TY, Chen TY, Chang KM, Chuang VP, Kao KJ. Plasmalemmal vesicle associated protein (PLVAP) as a therapeutic target for treatment of hepatocellular carcinoma. BMC Cancer. 2014;14:815.

21 Yokomori H, Oda M, Yoshimura K, Kaneko F, Hibi T. Aquaporin-1 associated with hepatic arterial capillary proliferation on hepatic sinusoid in human cirrhotic liver. Liver Int. 2011;31:1554-64.

22 Olsson AK, Dimberg A, Kreuger J, ClaessonWelsh L. VEGF receptor signalling - in con- trol of vascular function. Nat Rev Mol Cell Biol. 2006;7:359.

23 Tahir SA, Park S, Thompson TC. Caveolin-1 regulates VEGF-stimulated angiogenic activities in prostate cancer and endothelial cells. Cancer Biol Ther. 2009;8:2286.

24 Yokomori H, Ando W, Kaneko F, Suzuki H, Igarashi K, Oda M. Autotaxin and vascular endothelial growth factor receptor-2 and -3 are related to vascular development during the progression of chronic viral hepatitis $\mathrm{C}$. APMIS. 2018;126:913-21.

25 Hnasko R, Frank PG, Ben-Jonathan N, Lisant MP. PV- 1 is negatively regulated by VEGF in the lung of caveolin-1, but not caveolin-2, null mice. Cell Cycle. 2006;5:2012-20.

26 Pries AR, Kuebler WM. Normal endothelium. Handb Exp Pharmacol. 2006;176(Pt 1): $1-40$.

27 Strickland LA, Jubb AM, Hongo JA, Zhong F, Burwick J, Fu L, et al. Plasmalemmal vesicle associated protein (PLVAP) is expressed by tumour endothelium and is upregulated by vascular endothelial growth factor A (VEGF). J Pathol. 2005;206:466-75.

28 Tse D, Stan RV. Morphological heterogeneity of endothelium. Semin Thromb Hemost. 2010;36:236-45.

29 McCuskey RS. Morphological mechanisms for regulating blood flow through hepatic sinusoids. Liver. 2000;20:3-7.

30 Iguchi $\mathrm{H}$, Oda M, Yamazaki H, Yokomori $\mathrm{H}$. Participation of aquaporin-1 in vascular changes and remodeling in cirrhotic liver. Med Mol Morphol. 2013;46:123-32.

31 Roskams TA, Theise ND, Balabaud C, Bhagat G, Bhathal PS, Bioulac-Sage P, et al. Nomenclature of the finer branches of the biliary tree: canals, ductules, and ductular reactions in human livers. Hepatology. 2004;39:1739-45.

32 Clouston AD, Powell EE, Walsh MJ, Richardson MM, Demetris AJ, Jonsson JR. Fibrosis correlates with a ductular reaction in hepatitis C: roles of impaired replication, progenitor cells and steatosis. Hepatology. 2005;41: 809-18.
PLVAP Overexpression Is Related to Sinusoidal Angiogenesis in Liver Cirrhosis
J Vasc Res 2021;58:361-369

DOI: $10.1159 / 000516923$ 